\section{Comentario a propósito de la presentación de la Dra. Elza Berquó. Reflexiones teórico metodológicas sobre la elaboración de cuestionarios de encuesta: una experiencia de integración de enfoques}

\section{Comentário sobre a apresentação da Dra. Elza Berquó. Reflexões teórico-metodológicas sobre a elaboração dos formulários do inquérito: uma experiência de integração de abordagens}

\section{Irene Luppi}

Instituto de la Salud Juan Lazarte/Centro de Estudios Interdisciplinarios de la Universidad Nacional de Rosario. Argentina

\section{Resumen}

Se presenta una reflexión teórico metodológica sobre la elaboración de cuestionarios de encuesta recuperando dos cuestiones claves señaladas por la Dra. Berquó, en su testimonio sobre las iniciativas desarrolladas en el campo de la salud reproductiva con el objetivo de producir datos que se puedan utilizar para formular políticas e implementar intervenciones en los distintos contextos: la complejidad y la flexibilidad. En particular, cuando se abordan cuestiones de mayor dificultad para su objetivación, tales como los pareceres, las valoraciones de los sujetos en relación con sus prácticas y las experiencias de padecimiento y cuidado. Se enfatiza la necesidad de un riguroso examen de las categorías analíticas susceptibles de ser tratadas empíricamente y de la funcionalidad de los conceptos para la producción de indicadores de contenidos sistematizables para la comparación estadística. Se presenta una experiencia de aplicación de un cuestionario, como instancia de integración metodológica en una investigación por encuesta, destinada a lograr una aproximación directa al universo de representaciones y valoraciones de la mujer, no mediada por los códigos y la lógica de quien las indaga. Para su confección se recuperaron los hallazgos de una investigación antropológica preliminar y se armó un dispositivo de preguntas con expresiones textuales de las entrevistadas, a la manera de un esquema de apoyo del discurso de la mujer. La propuesta presentada, de relativa simplicidad, puede contribuir a superar dificultades vinculadas con la viabilidad de captación de determinadas temáticas según los contextos socioculturales, a nivel internacional.

Palabras clave: Cuestionarios de encuesta. Integración metodológica. Operacionalización de conceptos. 


\section{Resumo}

Trata-se de uma reflexão teórico-metodológica sobre a elaboração de formulários de inquéritos, recuperando duas questões fundamentais assinaladas pela Dra. Berquó em seu depoimento sobre as iniciativas desenvolvidas no campo da saúde reprodutiva, com o fim de produzir dados idôneos para a formulação de políticas e a implementação de intervenções nos diferentes contextos: a complexidade e a flexibilidade. Em particular, quando se abordam dimensões de maior dificuldade para sua objetivação, como os pareceres, as valorizações dos sujeitos em relação às suas práticas e experiências de padecimento e cuidado. Enfatizase a necessidade de um rigoroso exame das categorias analíticas suscetíveis de serem tratadas empiricamente, e da operacionalização dos conceitos para a produção de indicadores de conteúdos sistematizáveis para a comparação estatística. Apresentase uma experiência de aplicação de um questionário, como instância de integração metodológica em uma investigação por inquérito, destinada a conseguir uma aproximação direta do universo de representações e valorizações da mulher, não mediada pelos códigos e a lógica de quem as indaga. Para sua confecção foram recuperados os resultados de uma indagação antropológica preliminar e armado um dispositivo de perguntas com expressões textuais das entrevistadas, à maneira de um esquema de suporte do discurso da mulher. A proposta apresentada, de relativa simplicidade, pode contribuir para a superação de dificuldades vinculadas à viabilidade de captação de determinadas temáticas segundo os contextos socioculturais, no âmbito internacional.

Palavras-chave: Formulário de inquérito. Integração metodológica. Operacionalização de conceitos.

\section{Introducción}

El documento "Sobre el sistema internacional de investigaciones demográficas y salud reproductiva”, presentado por la Dra. Elza Berquó en el "Seminario Investigaciones poblacionales: aspectos metodológicos, operacionales y éticos" (ABRASCO, USPI, FCMSCSP, abril 2007), ofrece un detallado testimonio de las iniciativas realizadas desde la década del setenta ante la ausencia de datos confiables y la necesidad de producir información sobre niveles, tendencias y estándares de fecundidad esenciales para la formulación de políticas y la implementación de intervenciones en salud reproductiva. Esta preocupación ya se había instalado, desde la década anterior, en especial en los países del norte, ante el auge de la denominada “explosión demográfica” que llevó a los organismos internacionales a estimular la producción de datos tratando de llenar el vacío de estadísticas vitales internacionalmente comparables. ${ }^{1,2}$

Los esfuerzos destinados a la elaboración de un cuestionario básico en el marco de la Investigación Mundial de Fecundidad (WFS), "el mayor programa internacional de encuesta hasta entonces realizado", que se llevó a cabo en 62 países entre 1974 y 1984, muestran la complejidad de la tarea propuesta, con la participación de renombrados demógrafos y con un numeroso plantel de profesionales de reconocida experiencia y conocimiento.

Una de las dificultades mencionadas está relacionada con la viabilidad en los diferentes países para tener en cuenta temas, no sólo complejos para su definición conceptual y operacional, a los fines de su inclusión en un cuestionario de encuesta de alcance internacional, sino fundamentalmente delicados en términos políticos,

\footnotetext{
1 Comissão de População das Nações Unidas e Agência Americana para o Desenvolvimento Internacional (USAID), 1971. ${ }^{2}$ Conferencia da União Internacional para o Estudo Científico da População (IUSSP). Nueva York, 1961. Comitê de Estúdios Comparativos de Fecundidade y Planejamento Familiar, 19651967: "Variáveis para Estudos Comparativos de Fecundidade" (Cleland, 1987; Naciones Unidas: "Variáveis e questionários para análises comparativas", 1969.
} 
con potencial riesgo para la viabilidad de realización de la propia encuesta. Los debates suscitados en este sentido fueron superados apelando a la "flexibilidad en la aplicación, conforme al contexto". ${ }^{3}$

Es precisamente este rasgo de flexibilidad de los temas a incorporar lo que caracterizó los estudios iniciados en 1984, las encuestas de Demografía y Salud (DHS). Las mismas trataron de adaptarse, en sus sucesivas fases a los cambios sociales y a las necesidades específicas de los diferentes países, posibilitando abordar tanto nuevas dimensiones como visiones de acuerdo con los avances en el área (la indagación con hombres, HIV, papeles de género) así como el diseño de módulos adicionales sobre temáticas seleccionadas de inclusión según el interés de los países. ${ }^{4}$

Las investigaciones de este tipo en Brasil, el Estudio Nacional de Salud MaternoInfantil y Planificación Familiar (PNSMIPF1986) y el posterior Estudio Nacional de Demografía y Salud (PNDS-1996 y PNDS2006) incorporaron a su vez una potencialidad analítica, por estar basadas en diseños de muestras que sirvieron para obtener estimaciones independientes por regiones geográficas del país. ${ }^{5}$

Esta diferenciación permite avanzar en la comprensión de los determinantes y condicionantes de los perfiles de salud/ enfermedad de los grupos poblacionales teniendo en cuenta las condiciones sociales y materiales de vida y la organización social y política de los espacios donde se

${ }^{3}$ Ver referencia Lille, 1987 citada por Berquó.

${ }^{4}$ Con apoyo de USAID, Institute for Resource Development, Inc (IRD/Westinghouse Eletronic Company (1984)/Macro International Inc. (1989); MEASURE (Monitoring and Evaluation to Assess and Use Results), USAID: MEASURE DHS+ (1997 2002), MEASURE (2a fase, $2003-\ldots$ ).

${ }^{5}$ PNSMIPF 1986: BENFAM, Instituto para o Desenvolvimento de Recursos (IRD)/Westinghouse Eletric Company. Centro de Controle de Doenças (CDC), Atlanta, Estados Unidos de Norte América. PNDS 1996: BEMFAM, Macro International, Coordenação de Saúde da Mulher, do Adolescente e da Criança - COSAM/COSAD, Instituto Nacional de Alimentação e Nutrição - INAN, do Ministério da Saúde, da MACRO, do FNUAP, do UNICEF e da USAID. PNDS 2006: Cebrap, Núcleo de Estudos de População - NEPO/Unicamp Departamento de Medicina Preventiva da Faculdade de Ciências Médicas da Unicamp Laboratório de Nutrição do Departamento de Clínica Médica da Faculdade de Medicina de Ribeirão Preto, da Universidade de São Paulo, Núcleo de Pesquisas em Nutrição e Saúde da Faculdade de Saúde Pública da Universidade de São Paulo. realizan las intervenciones, las que aportan significativamente a la comprensión de los procesos sociales que contribuyen positiva y negativamente en la producción de salud $^{1-3}$.

\section{Sobre la complejidad de elaboración de cuestionarios de encuesta}

La realización de estudios de base poblacional son oportunidades significativas para desarrollar metodologías y contenidos sociodemográficos y epidemiológicos en sintonía con las necesidades de salud de la población.

En la preocupación por promover una reflexión en ese sentido interesa destacar la complejidad que supone elaborar cuestionarios de encuesta, en particular cuando se abordan temáticas de difícil objetivación como las referidas en la presentación de la Dra. Elza Berquó: percepciones, preferencias, intenciones de los entrevistados respecto de prácticas y orientaciones vinculadas con su salud sexual y reproductiva, el registro de los papeles y de los derechos sociales desde una perspectiva de género, entre otras.

En la práctica de indagación es posible encontrar un cierto sesgo en relación con esta tarea, que no es exclusivo del campo de la salud y que lleva a reificar la dimensión técnica y operativa en detrimento de la construcción teórico metodológica que sustenta el diseño de todo instrumento de captación de información.

La confección de un cuestionario de encuesta requiere de un trabajo conceptual que implica la definición minuciosa del significado y el alcance de los términos a emplear, su capacidad para traducir categorías analíticas susceptibles de ser tratadas empíricamente y la funcionalidad de los conceptos, que responde a los objetivos prácticos de producción de indicadores de contenidos sistematizables para la comparación estadística.

No deja de ser frecuente, desde posiciones empiristas o positivistas, atribuir los conceptos (o no observables) a la teoría y reservar la metodología a los modos de 
encontrar, formular $\mathrm{u}$ operar con los observables o indicadores de esos conceptos. Sin embargo, ambos componentes del proceso de trabajo de indagación, si bien pueden ser distinguibles analíticamente, de manera epistemológica están intrínsecamente vinculados, ya que los observables (indicadores) deben reemplazar válidamente a los no observables (conceptos) ${ }^{4}$.

Estos conceptos son abstracciones realizadas sobre la base del examen de lo real, no son simples definiciones o convenciones terminológicas sino que buscan alcanzar la realidad a partir de una selección, basada obviamente en conocimientos previos, que pretende rescatar lo que es esencial a los investigadores según la finalidad y objetivos de la propuesta.

Las definiciones adoptadas son claves porque además de orientar el cuestionario, fijarán los límites y alcances para la interpretación de la información a obtener. Para ello no sólo es necesario identificar las dimensiones principales involucradas, en relación al conjunto de fenómenos al que se aplica el concepto y al conjunto específico que se abordará a nivel empírico, en un espacio y tiempo determinados, sino que se requiere una clarificación previa del lenguaje enlazada con un trabajo de vigilancia epistemológica y metodológica para explicitar los presupuestos, las nociones (los conceptos previos) presentes en el habla, de modo que podamos hacer conciente cuál es la opción que se está tomando y cuáles son sus consecuen$\operatorname{cias}^{4}$.

Esta tarea se complica cuando en el espacio de una encuesta domiciliar se pretende recuperar aspectos vinculados con los niveles simbólicos de los problemas de salud/enfermedad de mayor dificultad para su objetivación, los pareceres, las valoraciones de los entrevistados en relación a sus prácticas y experiencias previas de padecimiento y de cuidado.

Un aspecto que amerita ser considerado es que la presumible ausencia de reflexión previa sobre los temas abordados dificulte la posibilidad de tornar tales elementos concientes y comunicables al encuestador. En otros términos, las respuestas estarán mediadas por las capacidades individuales de organizar, sintetizar y expresar verbalmente percepciones y representaciones sobre los mismos en el tiempo destinado a un cuestionario de encuesta.

A su vez, cuando se aplica un cuestionario, aún cuando el mismo esté reglado y acotado por las pautas de captación, no se puede ignorar que se trata de un espacio de interacción entre individuos, encuestadorencuestado, en la que se establece una relación interpersonal, que como tal, estará gobernada por valores y normas socialmente definidos en los contextos donde tiene lugar. Valores, expectativas, creencias previas vinculadas con los tópicos abordados, con lo que se presume debe ser respondido, pueden jugar un papel significativo al referir las prácticas indagadas y las apreciaciones en torno a ellas.

Es posible reconocer una cierta relación de asimetría en tanto es el encuestador quien conduce la interrogación, según una lógica impuesta en la organización del cuestionario y al mismo tiempo, la posibilidad de sesgos producidos por razones de orden cultural que explican por qué los conjuntos sociales estratificados definen sus problemas de salud/enfermedad, sus necesidades y acciones a través de lógicas diferenciales entre sí y diferenciales de las lógicas de las instituciones de salud.

Se debe destacar el aporte de las ciencias sociales para abordar cuestiones de este orden. No obstante, su articulación con otras áreas del conocimiento, si bien diversifica y amplía las posibilidades de los objetos de trabajo, al mismo tiempo entraña una complejidad creciente de los enfoques conceptuales que dificultan la construcción de métodos capaces de operacionalizarlos ${ }^{5}$.

\section{Experiencia de integración de abordajes cualitativos y cuantitativos}

Estas preocupaciones fueron objeto de cuestionamientos en una línea de investigación realizada en Rosario, Argentina, des- 
tinada fundamentalmente a proporcionar elementos para la elaboración de estrategias de cambio de los servicios de salud locales. El problema se definió ante la existencia de un posible nivel de ineficacia de los servicios de salud que estaría vinculado a desajustes en la relación entre los grupos poblacionales y agentes de salud. Al mismo tiempo se buscó llevar a cabo una propuesta que, tanto en términos conceptuales como metodológicos, permitiera poner a prueba perspectivas superadoras de los abordajes más generalizados dentro del complejo campo de análisis de la problemática de los problemas de salud/enferme$\mathrm{dad} /$ atención $^{6}$.

Se abordaron en un primer nivel las representaciones y prácticas orientadas a la solución de problemas de salud/enfermedad percibidos por la población femenina, a la vez que las representaciones que las mismas mujeres construyen alrededor de tales problemas y de la atención recibida. En un segundo nivel se intentó recuperar la perspectiva de los profesionales alrededor de las prácticas de atención de la población a su cargo ${ }^{7,8}$.

En el estudio con las mujeres, se consideró el problema de las dificultades que imponen las necesidades de objetivación y comparación propias de los métodos de producción de información estadística para una correcta recuperación de problemas complejos con variables que eluden una definición precisa.

En la convicción de que los conceptos que serán aplicados por los investigadores deben corresponderse con las formas en que los entrevistados piensan y actúan, con el objetivo de obtener información fidedigna sobre sus representaciones y prácticas fundadas en sus condiciones de vida y experiencias concretas, se vislumbró la posibilidad de articular enfoques, cuantitativos y cualitativos, para la elaboración del instrumento de captación de información, buscando un mejor abordaje y conocimiento del problema, desde la perspectiva de los sujetos.

Se quiso lograr una aproximación di- recta al universo de representaciones y valoraciones de la mujer, no mediada por los códigos y la lógica de quien las indaga, para lo cual se planteó un diseño de estudio diferenciando momentos de potencial integración de ambas lógicas: la del investigador y la del entrevistado, en este caso la mujer.

En primer lugar, se planteó la delimitación conceptual del problema y la identificación de categorías susceptibles de ser operadas para recuperar referentes empíricos según los objetivos propuestos, espacio privativo del equipo de investigación.

El trabajo de campo abarcó dos etapas. La primera consistió en un estudio antropológico, de tipo exploratorio, con entrevistas en profundidad a un grupo reducido de casos, dado que este tipo de entrevista facilita respetar la lógica de los entrevistados. A continuación, en el análisis del material reunido se reconstruyeron las categorías de los sujetos y en una primera instancia de integración, se buscó su articulación con los conceptos iniciales de los investigadores.

De este modo, los hallazgos más significativos de la primera etapa fueron tomados como punto de partida para la segunda, un estudio por encuesta a una muestra aleatoria de 629 mujeres residentes en un área de la ciudad de Rosario.

Se consideró el cuestionario como el elemento estratégico, tanto como instancia de articulación de las lógicas presentes como de integración metodológica.

La información obtenida en el estudio antropológico se tomó como base para la sistematización de los contenidos, su operación en términos de variables y transformación en preguntas para un cuestionario estructurado.

En alguna medida esta propuesta se inspira, en una perspectiva de triangulación metodológica, en las técnicas RAP (rapid assesment procedures), destinadas a obtener información en un tiempo relativamente corto, sobre creencias y percepciones de problemas de salud y de utilización de ser- 
vicios de atención. Provenientes de la antropología social en salud, su aplicación en base a guías con un listado de temas adaptados a las realidades locales, no requiere de conocimientos avanzados en métodos antropológicos. Basta con capacitar entrevistadores con experiencia en trabajo de campo y habilidad para desarrollar un buen nivel de comunicación con el entrevistado.

En común con los RAP se elaboró un instrumento con una serie de preguntas, las que por su sencillez y por incorporar expresiones textuales de las mujeres (de la primera etapa) permitieron anular la extrañeza que conlleva la aplicación de un cuestionario de encuesta, y actuaron formando un esquema de apoyo del discurso de la mujer al responderlas. Asimismo, tuvo como objetivo estimular la reflexión sobre la propia experiencia, expresarse en forma más libre, evitando sugerir o presionar, teniendo en cuenta que es posible inducir a la falsedad o incluso a la no respuesta como vía de escape ante una postura inquisitoria.

La tarea más compleja sin duda fue encontrar un criterio de sistematización que permitiera, a la luz de los resultados de mayor significación de la primera etapa, reconstruir las categorías planteadas inicialmente y llegar a una delimitación de los contenidos temáticos a incluir en el cuestionario y su traducción en preguntas. Llegado a este punto, y para cada una de las dimensiones identificadas en relación a una determinada categoría, se elaboraron listados de fragmentos de discurso vinculados con ellas y se seleccionaron varias expresiones verbales que fueron incluidas, sin respetar orden alguno, como alternativas de respuesta. La pregunta también fue formulada con la locución de utilización más generalizada y se armaron secuencias consistentes en una pregunta abierta seguida de una o varias preguntas cerradas.

El recurso de plantear múltiples alternativas apelando a un lenguaje sencillo, recuperado textualmente en el cestudio preliminar, fue adoptado para lograr una mayor consistencia de la información a recabar, estimándose que de esta manera se garantizaba una amplia comprensión de las preguntas por parte de las mujeres y su identificación con aseveraciones sentidas como de su propia vivencia.

Las secuencias de preguntas, abiertas y cerradas, se organizaron en capítulos según núcleos temáticos y nivel de registro, en una lógica de aproximación desde cuestiones menos invasoras y de carácter general, para avanzar gradualmente hacia esferas más privadas y complejas.

Las características peculiares del instrumento aumentaron las exigencias de capacitación para fortalecer el papel del entrevistador en el sentido mencionado, sin descuidar los criterios de uniformidad y replicabilidad propios de todo cuestionario estructurado. Se enfatizó el control lógico de consistencias, particularmente en todos aquellos puntos destinados a captar información de orden simbólico, con mayor dificultad de objetivación.

En particular, interesaba recuperar problemas relacionados con la esfera de la salud mental, vinculados con cuestiones emocionales, que habían sido expresados como "los nervios" y manifestado con importancia en el estudio antropológico de la primera etapa. Se buscaba aportar información sobre la problemática emocional reconocida por la mujer como malestar o perturbación en su vida cotidiana, no con fines de proporcionar un diagnóstico de las entidades nosológicas comprendidas, sino por el contrario, como una primera aproximación al problema. Se intentó verificar en qué medida este tipo de trastornos, en el nivel de inespecificidad en que son reconocidos, afectan a las mujeres y hasta qué punto los ubican dentro del área de competencia de la medicina, como para motivar una búsqueda de ayuda.

Se diferenciaron dos conceptos, referidos por la antropología médica en su idioma original para preservar en forma explícita su significado diverso: illness (malestar o perturbación de la vida cotidiana no inscriptos en el campo de intervención 
de la medicina) y disease (entidades nosológicas específicas desde la perspectiva médica).

Se preguntó si sufría alguna de las situaciones siguientes: no puede dormir, tiene temores, no tiene ganas de juntarse con gente, se siente triste, está deprimida, siente nerviosismo, se siente angustiada. Se agregó como una última alternativa, si estaba afectada por algún problema de salud "culpa de los nervios".

El primer hallazgo a destacar es que una elevada proporción de mujeres (58\%) reconoció una y más de las alternativas propuestas (illness) y sin embargo pasaron por alto la vinculación con problemas emocionales (disease). En esa instancia esto fue señalado sólo por un $24 \%$.

Sólo más tarde, una vez avanzada en buena parte la formulación de preguntas, en la secuencia mencionada, cuando el cuestionario se centró en el problema de salud asumido por las mujeres como el principal, en un lapso previo, la proporción de mujeres que reconoció estar afectada por problemas emocionales (illness) como su vinculación con un padecimiento de salud específico (disease) aumentó al 46\%.

El interés específico en relación a estos hallazgos en el marco de la indagación realizada parte del supuesto que estas cuestiones se cristalizan como dificultades en la relación población servicios de atención. En tanto síntomas, no forman parte de las representaciones construidas en torno a los problemas de salud y por ende quedan excluidos de la práctica médica.

A su vez, son ilustrativos de la problemática de captación de información y de la evidente necesidad de ajustar los instrumentos a los tiempos y lógicas de respuesta de los encuestados. En este caso, la dificultad inicial de la mujer para reconocer problemas de salud vinculados con "los nervios” se sorteó al lograr interiorizarla a su ritmo y gradualmente en el tema, procurando recuperar su propio discurso.

En los distintos capítulos del cuestionario, la regulación de preguntas buscó respetar dicha lógica, en primer lugar para superar potenciales inhibiciones de la mujer que las llevara a eludir hablar sobre temas con un mayor grado de privacidad en una situación de encuesta. En segundo lugar, para propiciar una reflexión sobre aspectos, que por ser parte de la vida cotidiana, no forman parte de la conciencia reflexiva sino de la conciencia práctica de los sujetos en contextos particulares.

El dispositivo utilizado, basado en el empleo de un repertorio en aparente desorden de expresiones textuales como alternativas de respuesta ayudó a lograr una generalizada comprensión de las preguntas, a la vez que favoreció una mayor confiabilidad de las respuestas. También dio a las mujeres la posibilidad de identificar dichas expresiones como propias, cercanas a su experiencia, y facilitar así su comunicación. La formulación de la pregunta previa, en forma abierta, a su vez sirvió como control de consistencias y para evitar imponer una dirección a las respuestas, según la lógica del investigador, contribuyendo a una mayor validez de la información recabada.

\section{Consideraciones finales}

La propuesta presentada, de relativa simplicidad para aplicar a nivel local, no parece sencilla de extender a encuestas de alcance internacional, en las que se busca responder a requerimientos de comparación.

Las experiencias aportadas por la Dra. Elza Berquó tratan tal complejidad y la necesidad de buscar la flexibilización de los instrumentos de captación. También del desafío de abordar problemáticas específicas de los países así como considerar obstáculos vinculados con la viabilidad de su abordaje según los contextos socioculturales.

Para ello cabría definir conjuntos básicos de cuestiones para monitorear a escala internacional y realizar al mismo tiempo indagaciones particulares y pasibles de desagregaciones de menor nivel (geográficas, administrativas, por unidades de ges- 
tión) e incluso incorporar perspectivas orientadas a plantear hipótesis, sin buscar inferencias sustentables estadísticamente.

Estas indagaciones pueden proporcionar líneas de análisis importantes y al mismo tiempo favorecer el desarrollo metodológico y técnico para la producción de información destinada al análisis de las desigualdades en el proceso salud/enfermedad/atención de los grupos poblacionales desde una perspectiva de espacio, para valorar cómo los contextos sociales, en sus distintas escalas de agregación, afectan los resultados y los riesgos de salud individuales en un territorio ${ }^{10}$.

El reto mayor en un escenario de orden internacional, cuando se necesita aplicar los mismos instrumentos, o buena parte de sus contenidos, es eludir que las traducciones cambien el sentido de las preguntas del cuestionario de encuesta para garantizar la validez y confiabilidad de los instrumentos ${ }^{11}$.

Una contribución posible residiría en pensar dispositivos que procuren superar una visión segmentada del objeto de estudio, como si estuviera ordenado en problemas diferentes por el hecho de utilizar técnicas provenientes de perspectivas metodológicas diferenciadas. Apelar no a una fusión de abordajes sino a una superposición, acompañada de una necesaria evaluación de las limitaciones y alcances de las técnicas e instrumentos a utilizar según el problema y el contexto de realización del estudio. Eso no significa olvidar que la integración de referenciales de distinto orden, cuantitativos y cualitativos es subsidiaria del modo de concebir la realidad, de la relación sujeto y objeto de conocimiento y del problema particular que se aborde.

\section{Referencias}

1. Castellanos PL. Epidemiologia, saúde pública, situação de saúde e condições de vida. Considerações conceituais. In: Barata R.(org.) Condições de vida e situação de saúde. Rio de Janeiro: ABRASCO; 1997. pp. 31-75.

2. Barata RCB. Epidemiologia Social. Rev Bras Epidemiol 2005; 8(1): 7-17.

3. Czeresnia D, Ribeiro AM. O conceito de espaço em epidemiologia: uma interpretação histórica e epistemológica. Cad Saúde Pública 2000; 16(3): 595-617.

4. Borsotti C. Temas de Metodología de la investigación en ciencias sociales empíricas. Buenos Aires: Miño y Dávila; 2007.

5. Costa MCN, Teixeira MGLC. A concepção de "espaço" na investigação epidemiológica. Cad Saúde Pública 1999; 15(2): 271-9.

6. Luppi I, Belmartino S, Bloch C, Boggio G. Investigaciones evaluativas en servicios de salud. Desarrollo metodológico de un proyecto en curso. Cuadernos Médico Sociales 1993; 64: 31-54.
7. Belmartino S, Bloch C, Luppi I, Schapira M. Mujer y servicios de salud: una perspectiva crítica. Rosario: CESS/ AMR: 1996

8. Luppi I. El encuentro médico-paciente: ¿armonía o conflicto? Rosario: Editora de la Universidad Nacional de Rosario; 2001.

9. Scrimshaw S, Hurtado E. Rapid Assesment Procedures for nutrition and primary health care. Tokio: The United Nations University; 1987.

10. Luppi I, Boggio G. Los modelos de niveles múltiples: una estrategia analítica para el estudio de los problemas de salud de la población. Rev Bras Epidemiol 2006; 9(1): 4255 .

11. Nigenda G, Romero M, Langer A. Reflexiones metodológicas en torno a un estudio cuali-cuantitativo sobre la percepción de la calidad de la atención prenatal en países en desarrollo. In Gogna M, Ramos S. (comp.) Experiencias Innovadoras en Salud Reproductiva. Buenos Aires: Consorcio Latinoamericano de Programas en Salud Reproductiva y Sexualidad CEDES/COLMEX/NEPOUNICAMP; 2002. 